\title{
Interferometric multi-core fiber optic gyroscope under temperature changing environment
}

Shinji MITANI(1), Kenichiro NIGO(1),

Satoshi KARASAWA(2), Haruyuki ENDO (2) and Taketoshi TAKAHATA ${ }^{(2)}$

(1) Japan Aerospace Exploration Agency (JAXA)

(2) Optoquest Co. Ltd. 


\section{Contents}

- Introduction

- Operating principle and problems of I-FOG (Interferometric FOG)

- Proposed fiber coil using MCF (Multi-core fiber) and FIFOs (Fan-in/Fan-out)

- Advantages of using MCF and FIFO

- MCF and FIFO devices

- Fabricated fiber coil using 7-core MCF and FIFO

- Experiment and Its Results

- Experimental setup of the 7-core MC-FOG

- Performance of the 7-core MC-FOG

- Temperature dependency

- Conclusions 


\section{Introduction}

- Background and motivation

- Interferometric fiber-optic gyroscope (I-FOG) with a long length fiber (a few km, typically) is widely used in navigation grade application

$\checkmark$ Long length fiber and elaborate coil manufacturing process

cost increase

- A novel architecture using multicore fibers (MCFs) and fan-in/fan-out (FIFO) devices

- We can shorten the physical fiber length to that divided by the number of core, while maintaining its optical length.

- We fabricated the fiber coil by using a 102-m long, 7-core MCF and implemented it into an open-loop I-FOG system

- We confirmed its superior performance as a rotation sensor for the first time $\left({ }^{*}\right)$

- Temperature dependency (well- know error source) is confirmed to be simple for compensation

* : Mtani, Nigo, et al., Interferometric Fiber-Optic Gyroscope Using Multi-Core Fiber, one of TOP SCORE papers of ECOC2018(Rome), to be issued in the Journal of Lightwave Technology (Special issue) 


\section{Operating principle and problems of I-FOG}

Operating principle - A Sagnac Interferometer

1. The signal light (from the source) is divided by DC1, and the two divided beams propagate in the fiber coil in the CW and CCW directions. Then both beams are recombined by DC1

2. A relativistic phase delay occurs depending on the rotation rate, and the phase difference between both beams (in the $\mathrm{CW}$ and CCW directions) $\Delta \phi_{R}$ :

$$
\Delta \phi_{R}=\frac{2 \pi L D}{\lambda c} \cdot \Omega
$$

$L$ : length of fiber in the coil

$D$ : Diameter of the fiber coil

$\lambda$ : Wave length of light

$c$ : Speed of light in vacuum

3. The phase shift $\Delta \phi_{R}$ is proportional to the rotation rate $\Omega$ and can be measured by detecting the interference signal

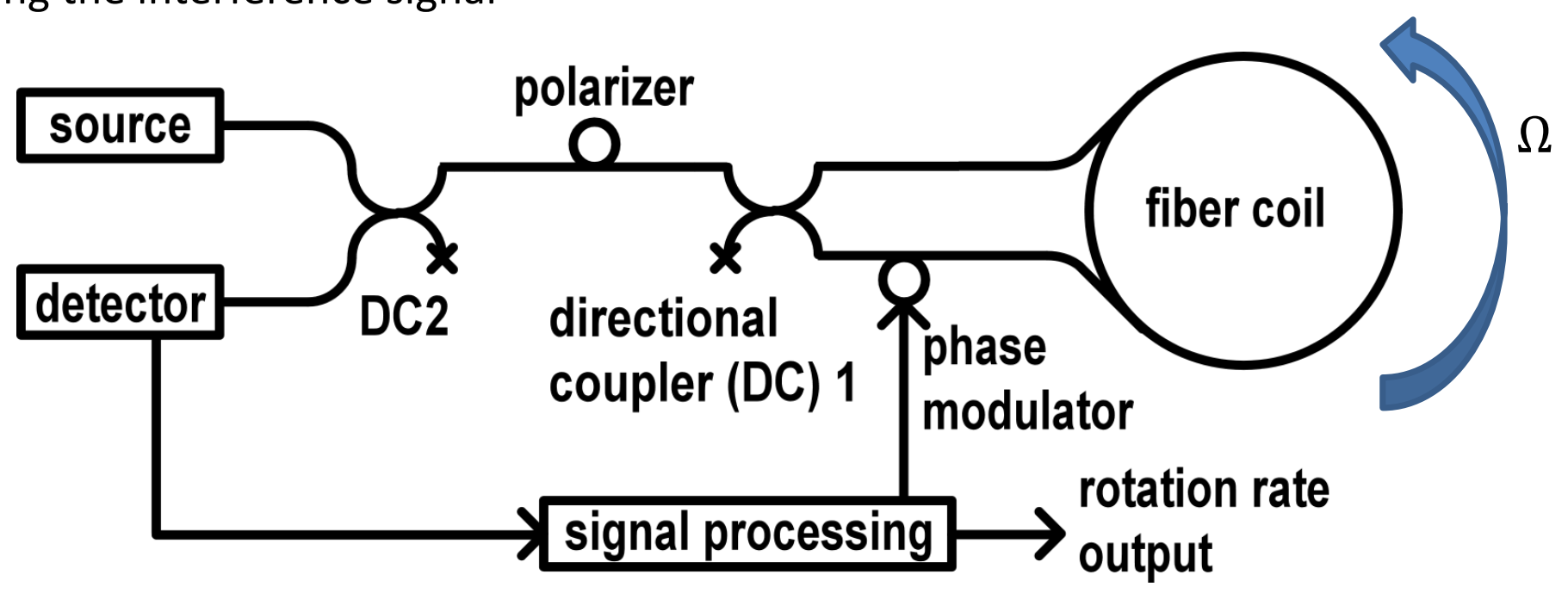

Fig. Schematic of conventional I-FOG configuration 


\section{Operating principle and problems of I-FOG}

- Objective

- Increase the fiber length $L$ to realize better sensitivity

- Key issues

- Shupe error suppression and manufacturing cost

$\checkmark$ A very meticulous winding process is timeconsuming

$\checkmark$ The long length fiber coil consequently increases the manufacturing cost

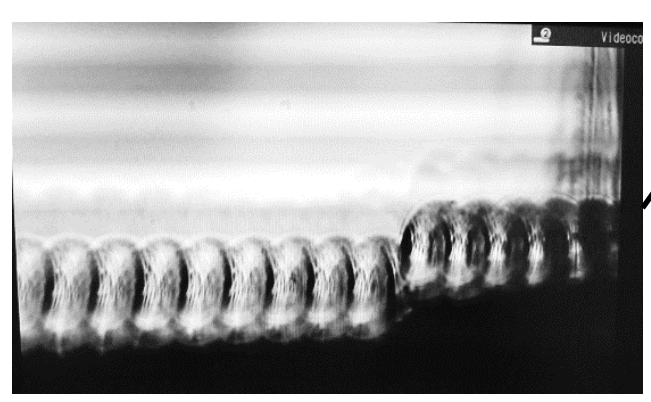

Fiber winding in magnified picture

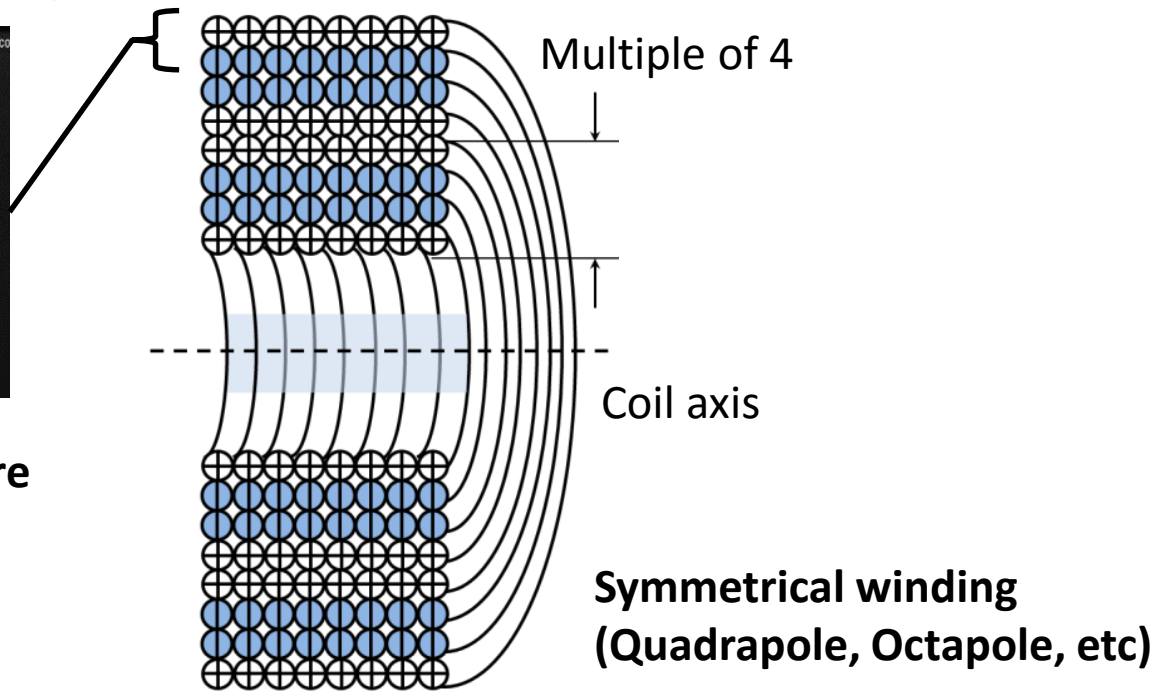




\section{Proposed fiber coil using N-core MCF and FIFOs}

\section{Basic Idea}

- Port $n$ of the FI device is connected to the port $(n-1)$ of the FO device $(n=$ $2, \cdots, N)$

- Port 1 of the FI device and port $N$ of the FO device are connected to DC1, and thus serve the role of input/output for the fiber coil

- In this way, an integrated single waveguide loop is realized by using FIFO devices, and the fiber coil's effective optical path length can be increased by a factor of $N$.

Fan-in (FI) port

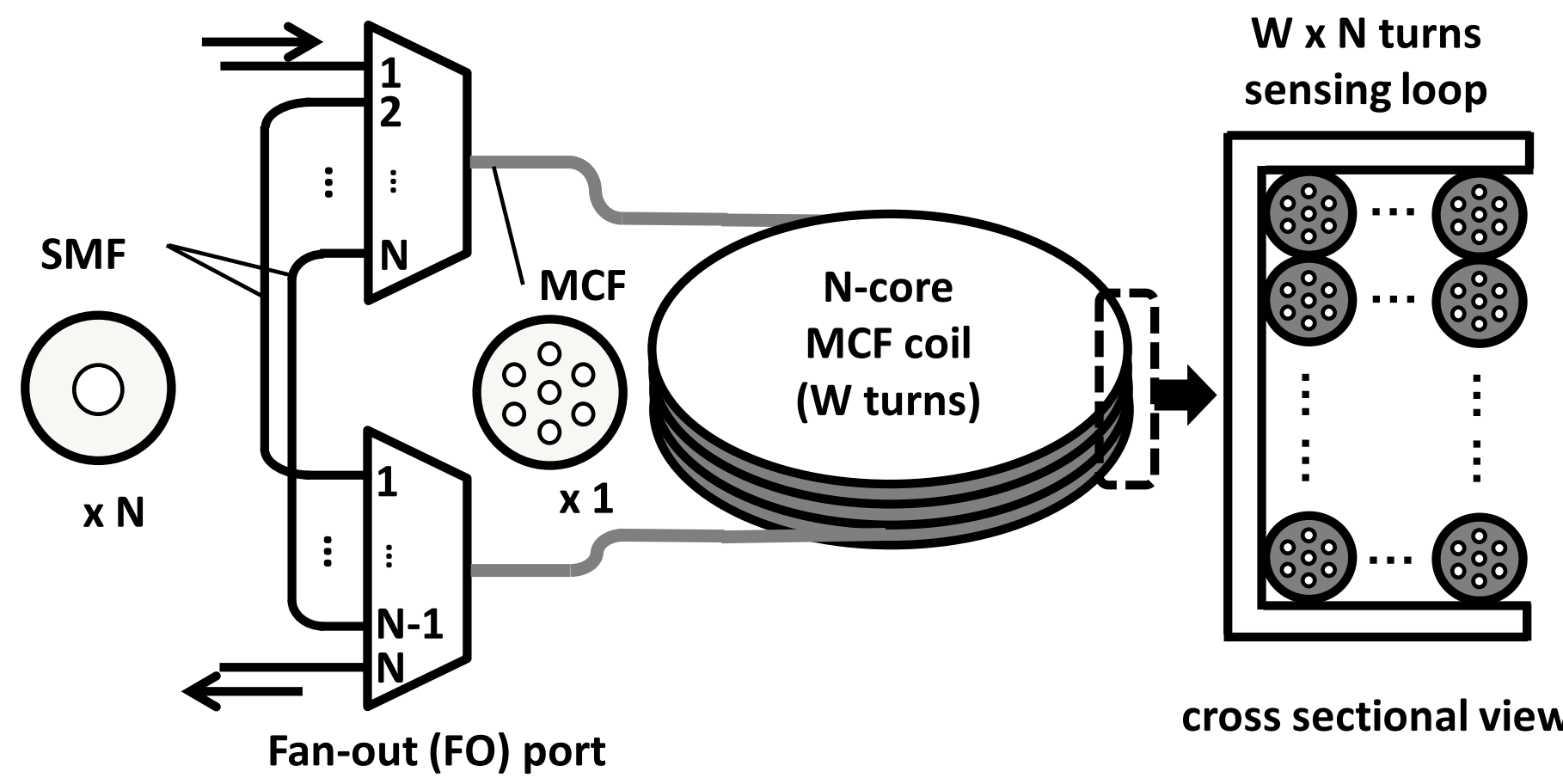




\section{Advantages of using MCF and FIFO}

- Using MCF in the I-FOG coil leads to many advantages:

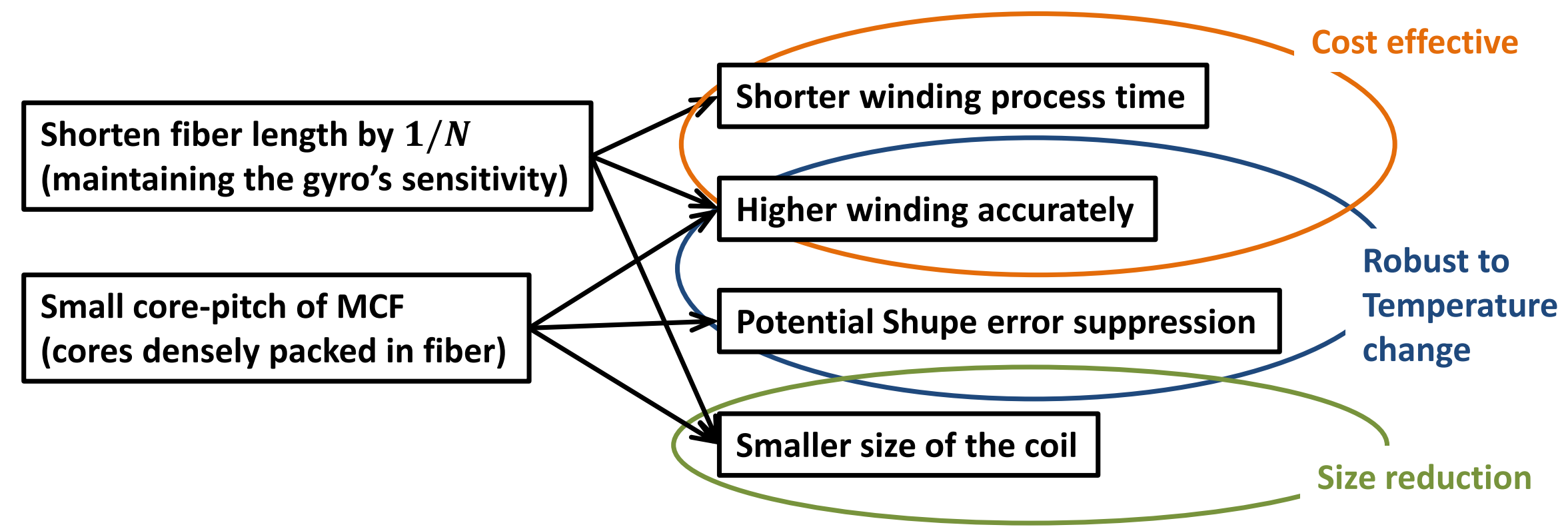

- Using FIFO in the I-FOG coil has following advantages:

- Selectability of core-to-core connection at the end of MCF in any order

- Applicability to any type of MCF with large number of cores and/or complicated core-arrangement 


\section{MCF and FIFO devices}

\section{Multi-core fiber (MCF)}

\begin{tabular}{|l|l|l|}
\hline Item & Specification & Note \\
\hline Core Number & 7 & \\
\hline Core Pitch & $45 \pm 1 \mu \mathrm{m}$ & \\
\hline Clad Diameter & $150 \pm 1 \mu \mathrm{m}$ & \\
\hline MFD & $10 \pm 1 \mu \mathrm{m} @ 1550 \mathrm{~nm}$ & \\
\hline Crosstalk & $\leqq-30 \mathrm{~dB} / \mathrm{km} @ 1550 \mathrm{~nm}$ & Between \#1 and \#5 \\
\hline Cutoff $\lambda$ & $\leqq 1530 \mathrm{~nm}$ & \\
\hline Loss & $1 \mathrm{~dB} / \mathrm{km} @ 1550 \mathrm{~nm}$ & \\
\hline
\end{tabular}

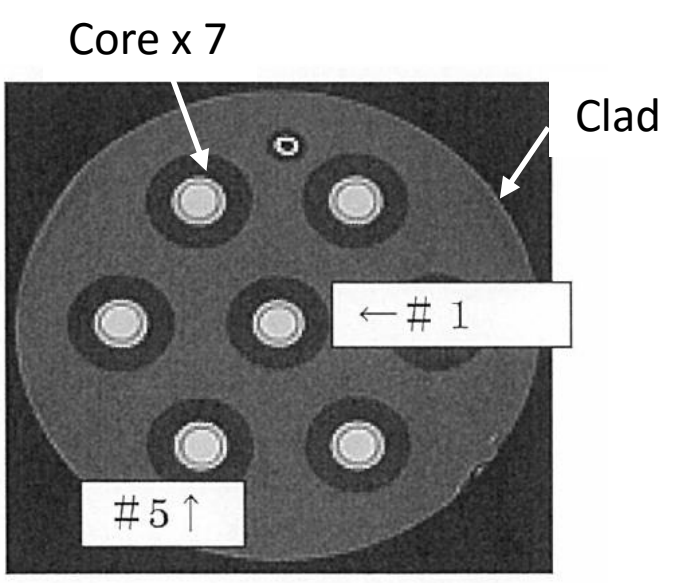

MCF core arrangement design (Hexagonal close-packed structure)

\section{Fan-in/Fan-out (FIFO) devices}

\begin{tabular}{|l|l|l|}
\hline Item & Measurement & Note \\
\hline Connection type & Free-space optics & Low loss \\
\hline Insertion loss & $<0.3 \mathrm{~dB}$ & All ports \\
\hline PDL & $<0.05 \mathrm{~dB}$ & All ports \\
\hline Return loss & $>50 \mathrm{~dB}$ & All ports \\
\hline
\end{tabular}

Fiber collimator $x 7$

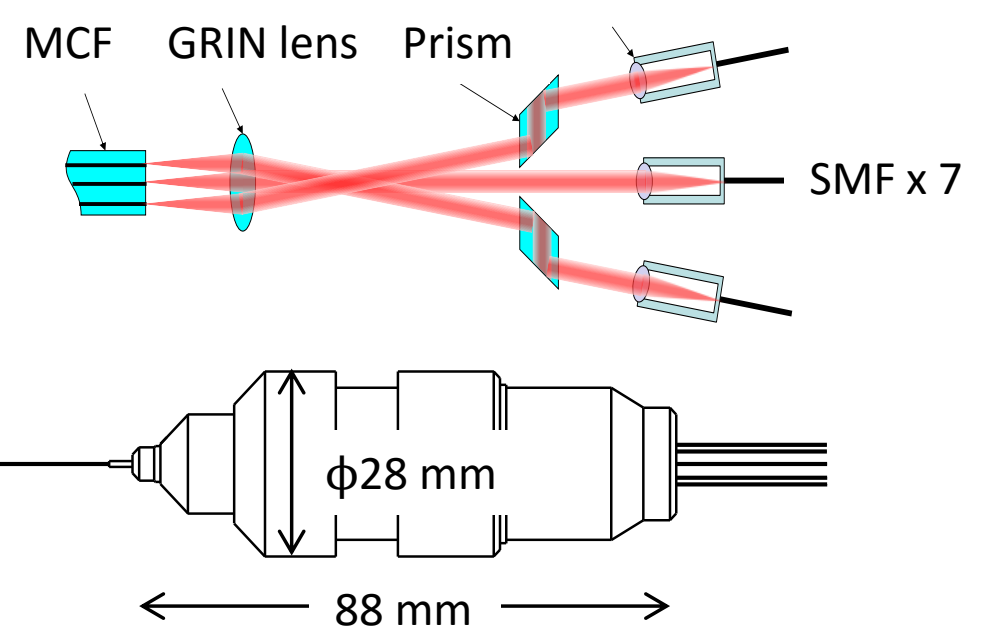




\section{Fabricated fiber coil using 7-core MCF and FIFO}

- A 102.7-m long MCF was wound on a titanium bobbin ( $\phi 128 \mathrm{~mm}$ )

- The total optical path length of the fiber coil was $\underline{823.8 \mathrm{~m}}$

- Symmetric winding pattern (Octupole winding) with a total of eight layers

- MCF + FIFO loop total performance :

\begin{tabular}{|l|l|}
\hline \multicolumn{1}{|c|}{ Item } & Result \\
\hline Insertion loss & $8.40 \mathrm{~dB}$ \\
\hline PDL & $0.05 \mathrm{~dB}$ \\
\hline
\end{tabular}

Return loss

FI side: $45.9 \mathrm{~dB}$

FO side: $48.0 \mathrm{~dB}$

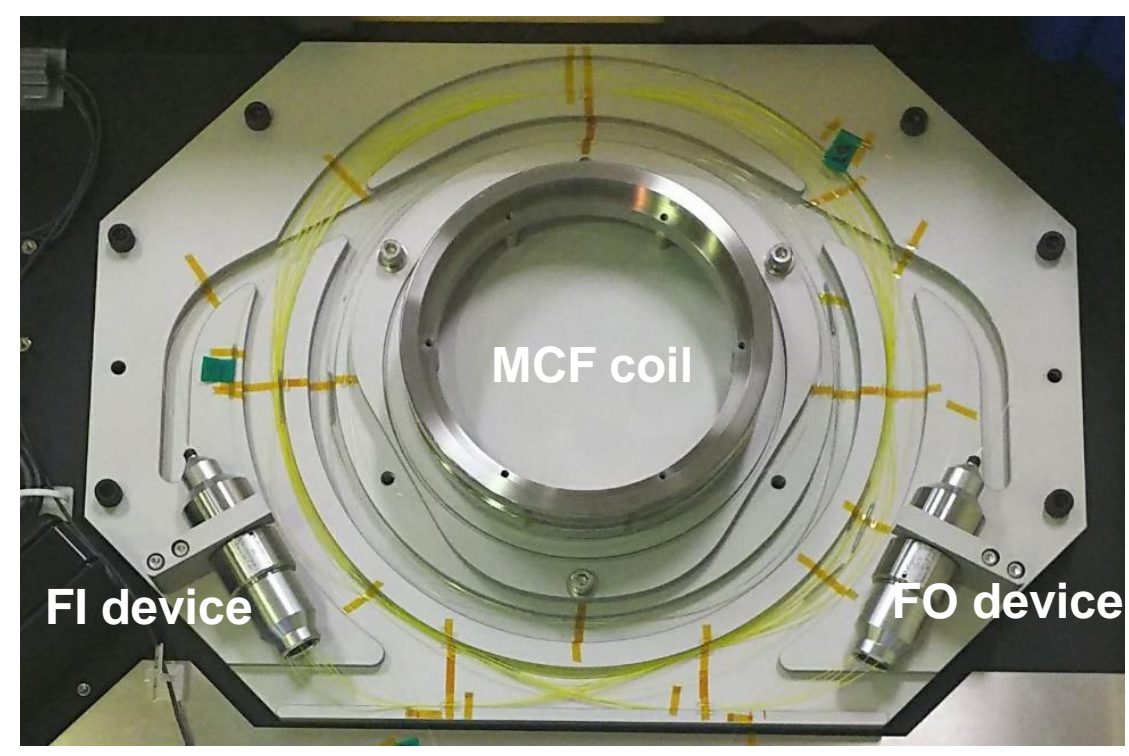

Fig. Fabricated fiber coil using 7-core MCF and FIFO

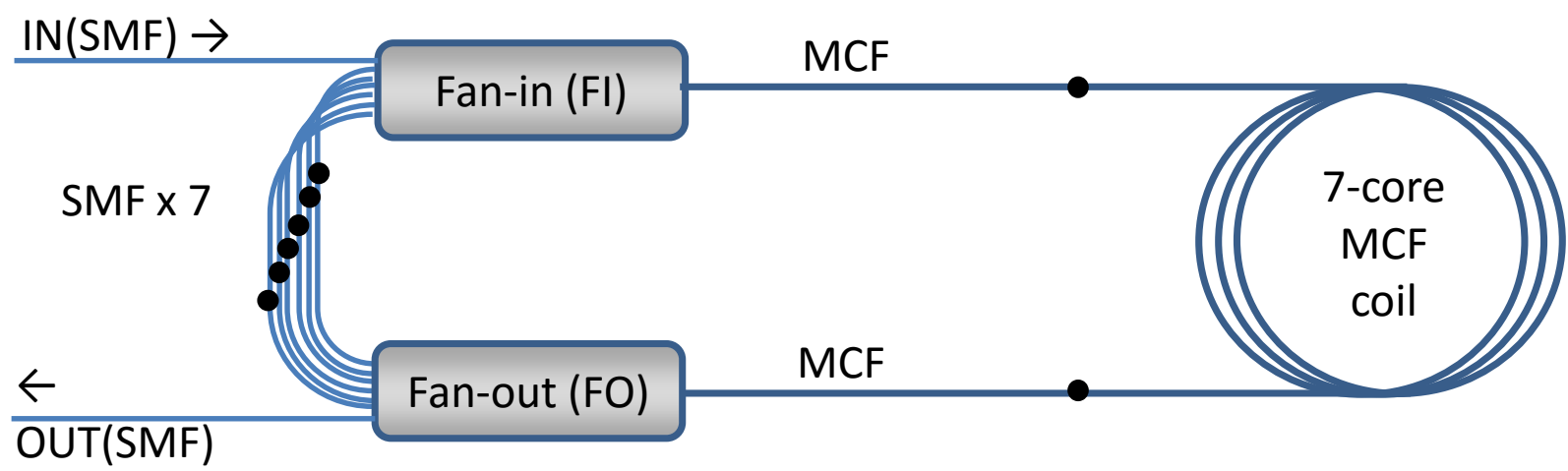

Fig. Schematic of the MCF and FIFO loop 


\section{Experimental setup of a 7-core MC-FOG}

- Open-loop FOG system

- $\quad$ SLD source (1550nm) with output power of $25 \mathrm{~mW}$ and a spectral width of $50 \mathrm{~nm}$

- Phase modulated with a sinusoidal signal at a frequency of $127.6 \mathrm{kHz}$

- The power received at the PD : $20.4 \mu \mathrm{W}$

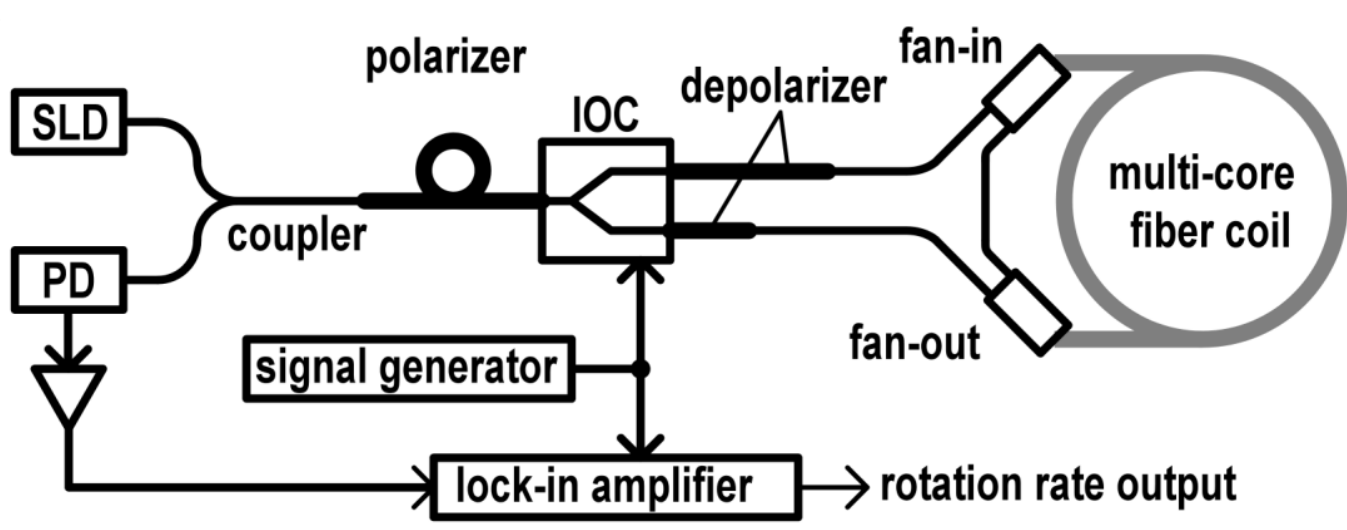

Fig. The implemented MC-FOG open loop system 


\section{Performance of the 7-core MC-FOG}

- The measurement result of the rotation rate

- The range of $\pm 10 \mathrm{deg} / \mathrm{s}$ (applied around the rate sensitive axis )

- The proposed system can sense the rotation rate linearly

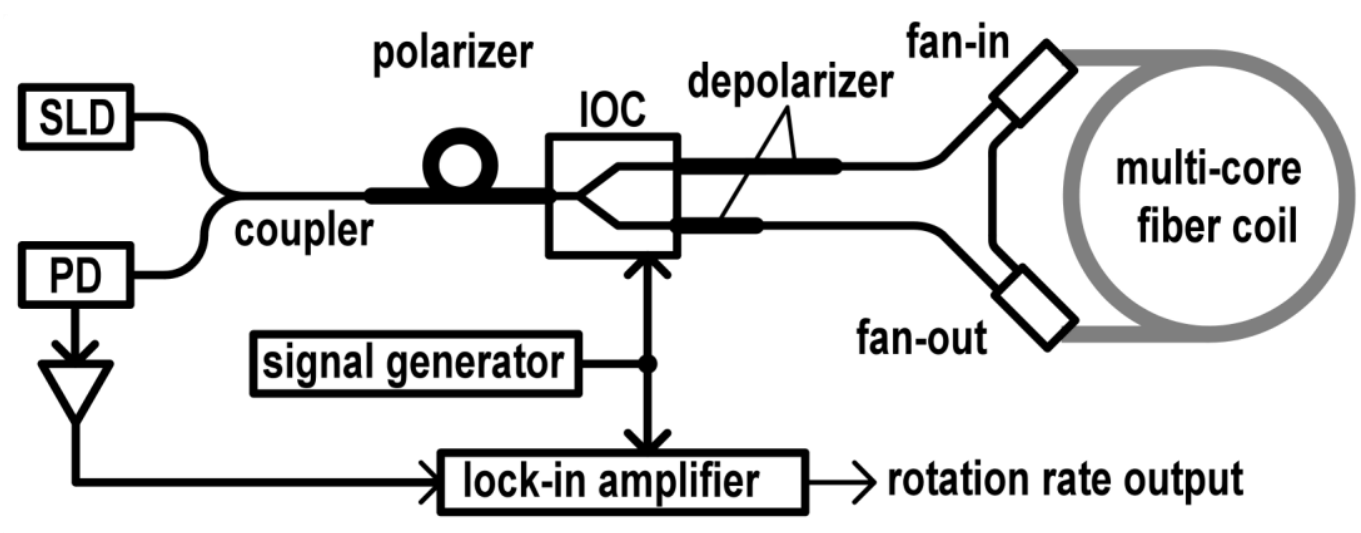

Fig. The implemented MC-FOG open loop system

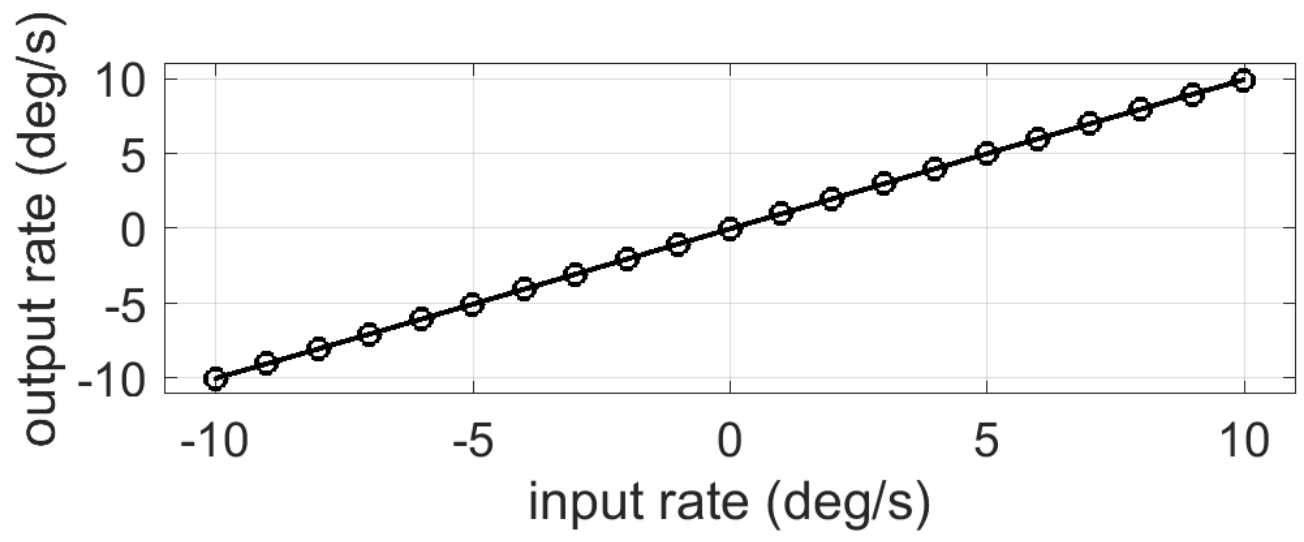

Fig. Measured rotation rate in the range of $10 \mathrm{deg} / \mathrm{s}$ 


\section{Performance of the 7-core MC-FOG}

- $\quad$ The measurement result for 12 hours statically

- The chambers' temperature : $25^{\circ} \mathrm{C}$

- Earth rotation rate $8.85 \mathrm{deg} / \mathrm{h}$ around the vertical axis

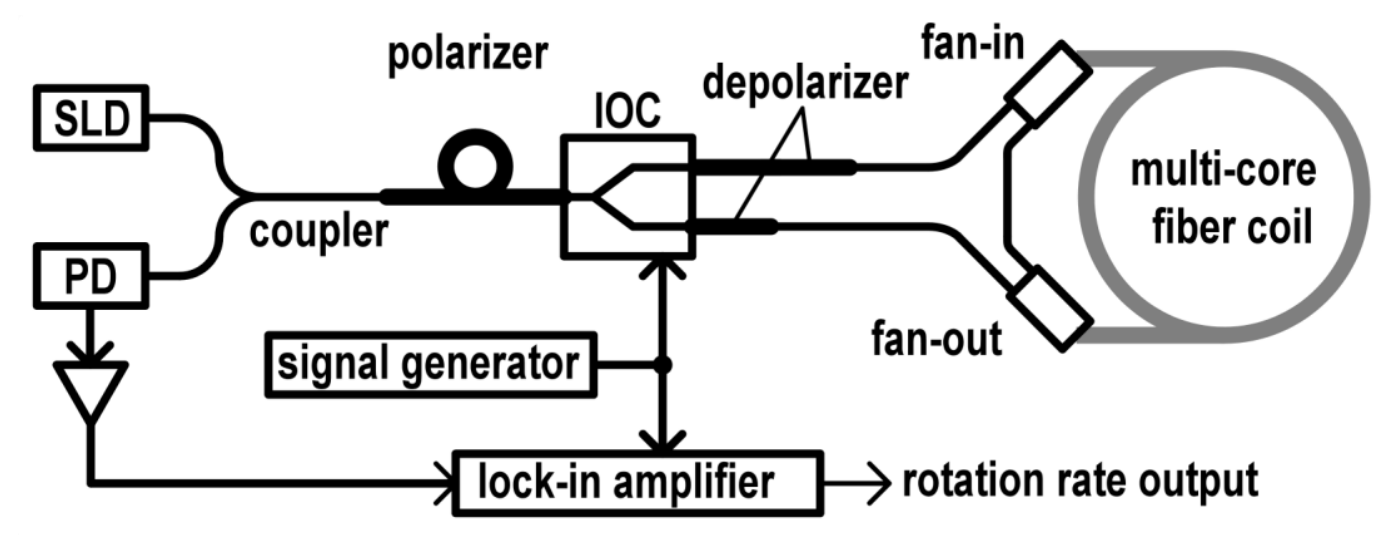

- Sufficient performance to sense the earth rotation rate

Fig. The implemented MC-FOG open loop system

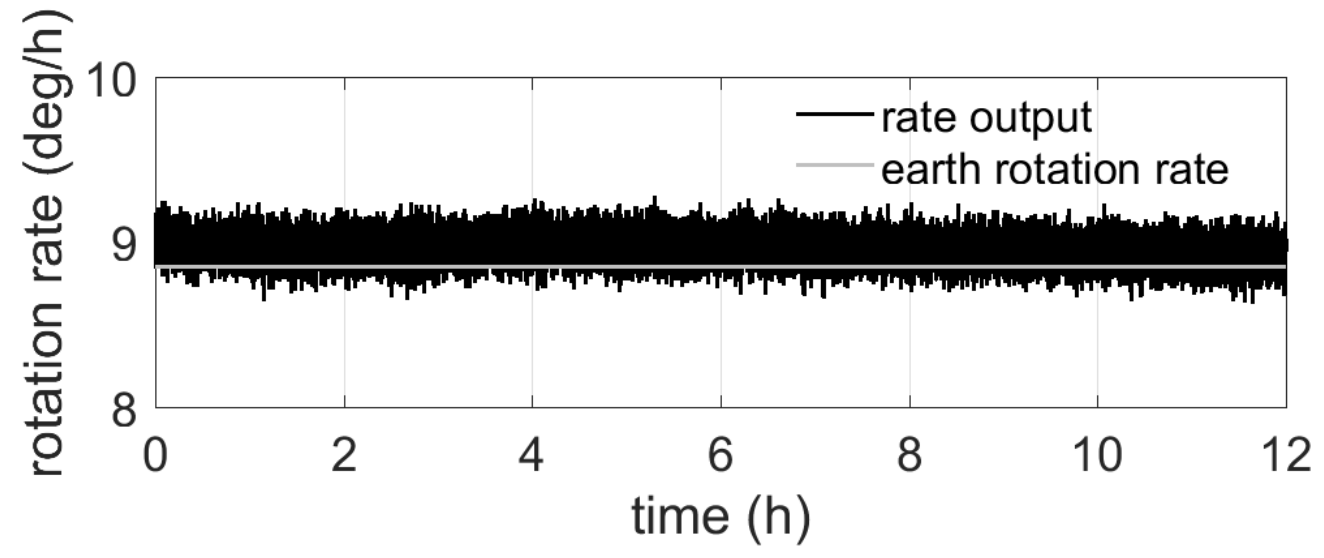

Fig. Output rate profile at $1 \mathrm{~Hz}$ sampling statically 


\section{Performance of the 7-core MC-FOG}

- The sensitivity close to that of the 700-m long fiber coil was achieved in spite of its physical fiber length of $102 \mathrm{~m}$.

- Clear proof of concept.

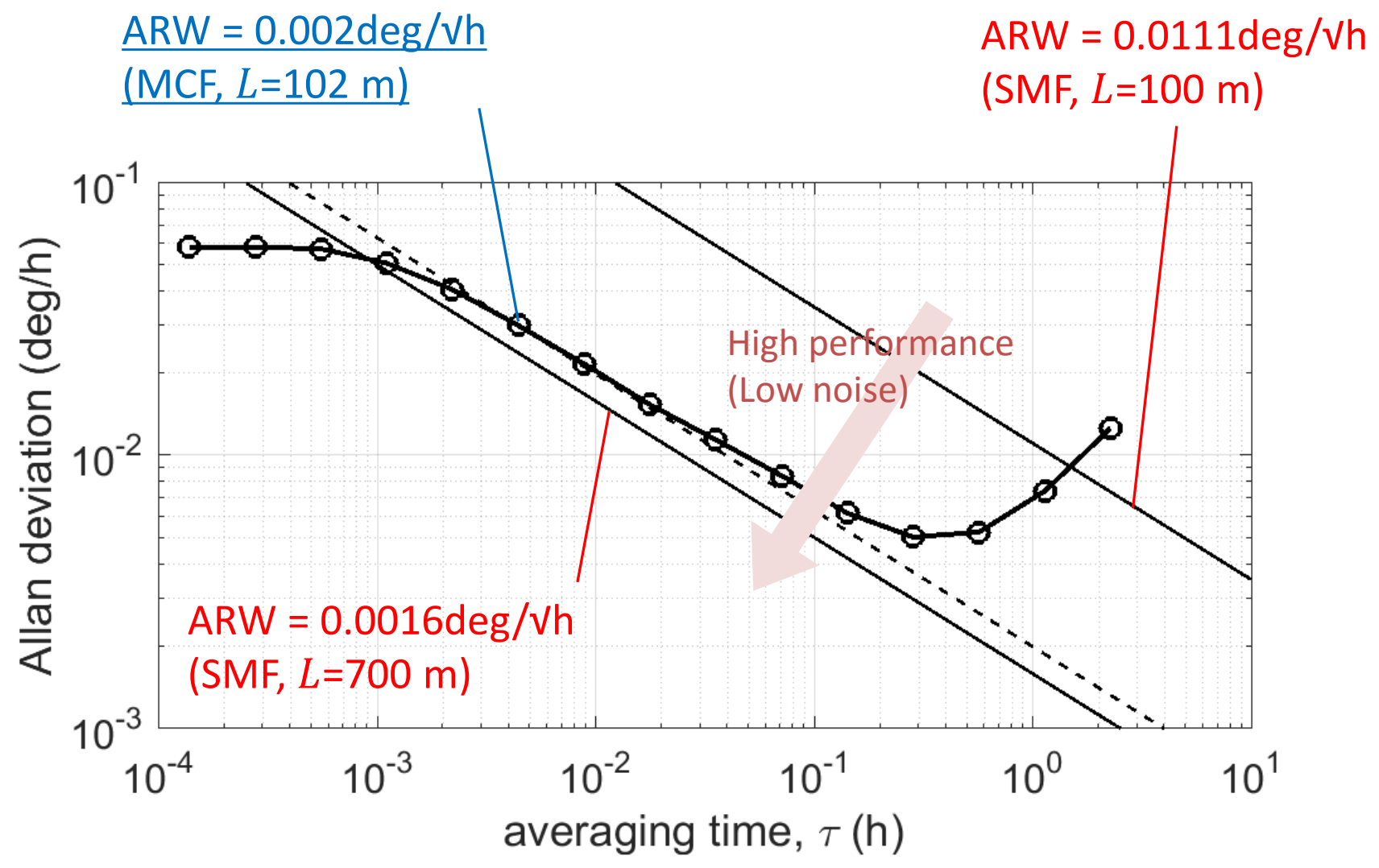

Fig. Allan deviation plot and theoretical ARW noise limit

[Note] Angle random walk (ARW): Gyro rate noise in short term which compose of the shot noise and excess RIN etc. 


\section{Temperature dependency Measurement}

- Thermally induced errors are one of the most dominant error cause in I-FOG.

- To confirm the feasibility of MC-FOG concept, we need to know the dependency of temperature of the newly introduced parts, i.e. MCF coil, Fan-in and Fan-out.

Thermocouple on the coil

Heater on FI

Thermocouple on FI

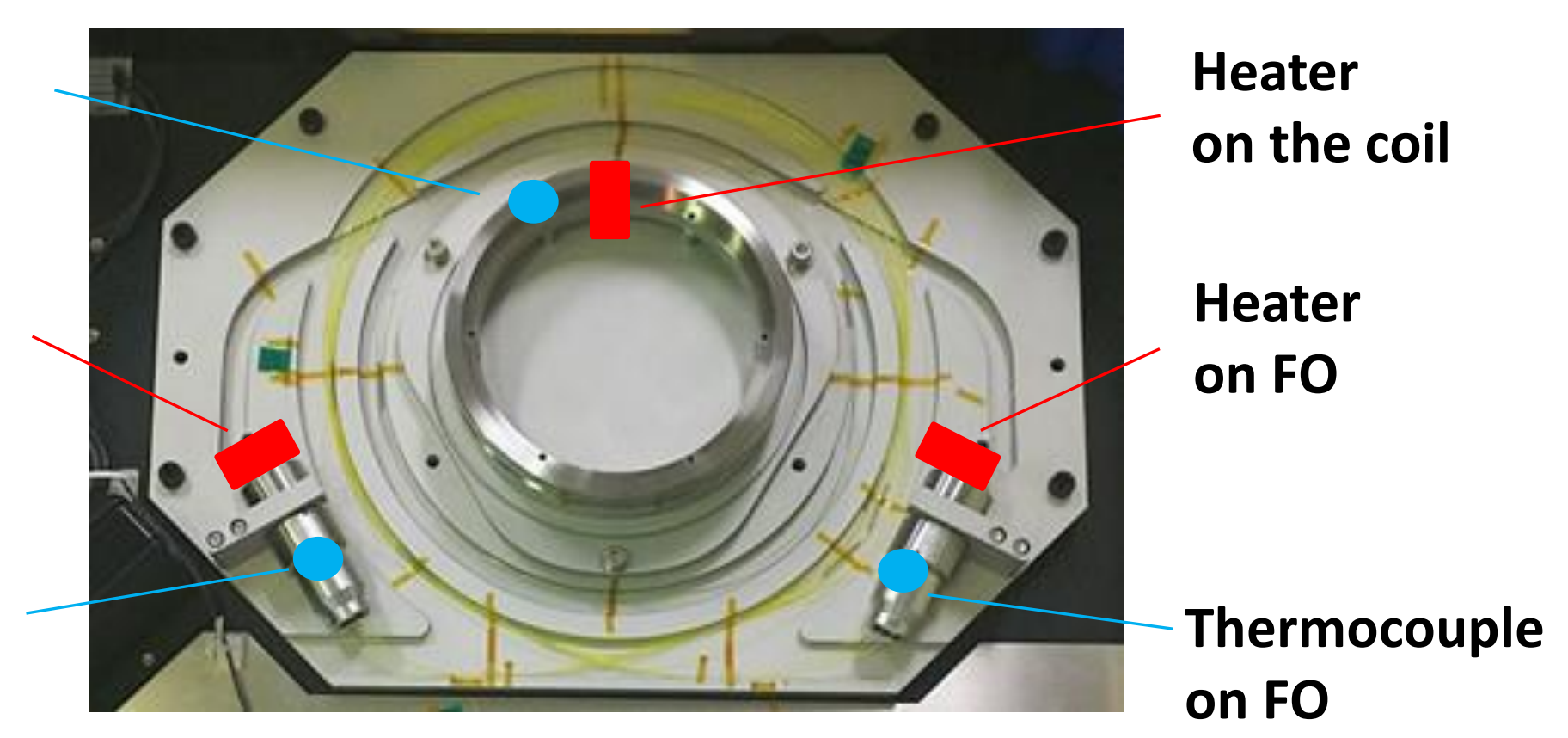




\section{Temperature dependency Measurement}
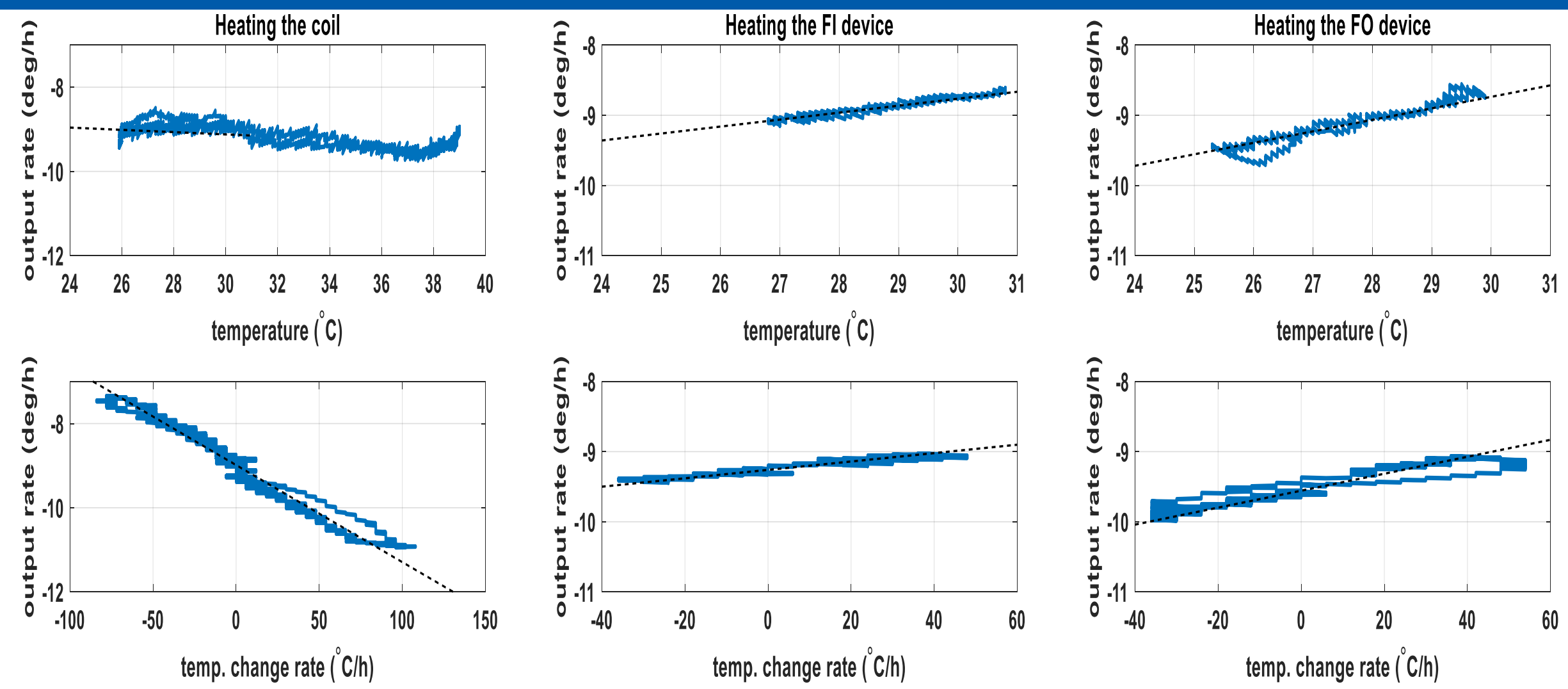

\begin{tabular}{|c|r|r|}
\hline $\begin{array}{c}\text { Location of } \\
\text { heat load }\end{array}$ & T slope $\left[(\mathrm{deg} / \mathrm{h}) /{ }^{\circ} \mathrm{C}\right]$ & \multicolumn{1}{|c|}{$\mathrm{dT}$ slope } \\
{$\left[(\mathrm{deg} / \mathrm{h}) /\left({ }^{\circ} \mathrm{C} / \mathrm{h}\right)\right]$} \\
\hline MCF Coil & -0.027 & -0.023 \\
\hline FI device & 0.099 & 0.0060 \\
\hline FO device & 0.16 & 0.012 \\
\hline
\end{tabular}

Shupe effect: A nonreciprocal phase shift caused by a timevarying distribution of temperature and stress in the fiber coil 


\section{Conclusions}

- The novel I-FOG sensor coil consisting of MCF spliced with FIFO devices was proposed

- The seven-core MCF coil with FIFO devices was fabricated

- And the operation of the proposed I-FOG was demonstrated for the first time

- The ARW performance of $0.002 \mathrm{deg} / \mathrm{Vh}$ was achieved, thereby confirming that the 7-core waveguide loop works successfully as a Sagnac interferometer as expected

- The dependencies on temperature conditions of MCF coil and FIFOs appear to be fairly simple and easy to be compensated: it is not anticipated to be a show-stopper for future MC-FOG development.

\section{OPTOQUEST}




\section{Conclusions}

\section{Future work to expand the potential of MC-FOG}

- MC-FOG with large core and extremely long loop

- Digital closed loop MC-FOG

- Smaller FIFO

- Developing polarization-maintaining MCF to improve the lowdrift performance

\section{Acknowledgment}

The authors are grateful to S. Nakamura, Y. Takushima (Optoquest Co. Ltd.), and T. Araki (JAXA) for lending their expertise on fiber optic gyroscope and laser optics through useful discussions.

\section{OPTOQUEST}

